\title{
Optical pulse propagation in metal nanoparticle chain waveguides
}

\author{
Stefan A. Maier, * Pieter G. Kik, and Harry A. Atwater \\ Thomas J. Watson Laboratory of Applied Physics, California Institute of Technology, Pasadena, California 91125
}

(Received 4 March 2003; published 6 May 2003)

\begin{abstract}
Finite-difference time-domain simulations show direct evidence of optical pulse propagation below the diffraction limit of light along linear arrays of spherical noble metal nanoparticles with group velocities up to $0.06 c$. The calculated dispersion relation and group velocities correlate remarkably well with predictions from a simple point-dipole model. A change in particle shape to spheroidal particles shows up to a threefold increase in group velocity. Pulses with transverse polarization are shown to propagate with negative phase velocities antiparallel to the energy flow.
\end{abstract}

DOI: 10.1103/PhysRevB.67.205402

PACS number(s): 78.67.Bf, 42.70.Qs, 42.82.Et, 71.45.Gm

Investigations of the interaction of light with metal nanoparticles have led to a wealth of knowledge about the fundamental electromagnetic processes occurring on length scales smaller than the wavelength $\lambda$ of light. ${ }^{1,2}$ Near-field investigations of the optical properties of single noble metal nanoparticles have confirmed the century old prediction that light at the surface plasmon resonance frequency interacts strongly with a single metal nanoparticle and excites a coherent collective electron motion, or plasmon. ${ }^{3,4}$ For a particle with a diameter $D \ll \lambda$, the light-matter interactions lead to an oscillating homogeneous polarization of the nanoparticle volume, resulting in an oscillating dipole field. For spherical $\mathrm{Au}$ and $\mathrm{Ag}$ nanoparticles in air, the dipole plasmon resonance frequencies lie in the visible part of the spectrum. ${ }^{1}$

Advances in particle synthesis and fabrication enable a study of the electromagnetic interactions between metal nanoparticles in ordered arrays with various particle spacings $d$. Such nanoparticle arrays exhibit collective dipole resonances. For $d \geqslant \lambda$, the collective dipole resonance frequencies and the lifetime of the plasmon oscillations are influenced predominantly by far-field dipolar interactions with a $d^{-1}$ distance dependence. ${ }^{5,6}$ For closely spaced metal nanoparticles $(d<\lambda)$, interactions mediated by the high local electromagnetic fields near the particle surface dominate with a $d^{-3}$ distance dependence. Far-field polarization spectroscopy on ordered linear arrays of closely spaced Au nanoparticles indeed showed a distance dependence of $d^{-3}$ of the interparticle coupling. 7,8

An intriguing application of the interaction between metal nanoparticles is the construction of waveguides for electromagnetic energy at visible or near-infrared frequencies. It was shown that far-field interactions between Au particles in a grating can establish a surface plasmon-polariton bandgap in analogy to photonic crystals, allowing for the construction of line defect waveguides. ${ }^{9}$ It has also been shown that the minimum size of these defect waveguides is determined by the diffraction limit $\lambda / 2 n$ of light due to the large spacing $d$ between adjacent particles that is necessary for the establishment of the bandgap.

Two recent publications have discussed the possibility of guiding electromagnetic energy with a lateral confinement below the diffraction limit of light in ordered onedimensional arrays of metal nanoparticles with interparticle spacings $d \ll \lambda$, using either extended Mie theory ${ }^{10}$ or a model which treats the particles as point dipoles. ${ }^{11}$ Interactions between adjacent nanoparticles in such plasmon waveguides were shown to set up coupled plasmon-polariton modes, which lead to coherent energy transport along the particle arrays. The point-dipole model allowed for the determination of the dispersion relation $\omega(\mathbf{k})$ of the plasmon modes which is shown in the inset of Fig. 1 for both longitudinal modes $L$ (electric field parallel to the chain axis) and transverse modes $T$ (electric field perpendicular to the chain axis) in the nearest-neighbor approximation. The group velocity $\nu_{g}$ for energy transport given by $d \omega / d \mathbf{k}$ is highest for the $\mathbf{k}=\pi / 2 d$ modes, which can be excited using local excitation at the plasmon resonance frequency $E_{0}$ of a single nanoparticle. Group velocities up to $10 \%$ of the velocity $c$ of light in vacuum were predicted. ${ }^{11}$ Also, a variety of different guiding geometries such as $90^{\circ}$ corners and tee structures as well as an all-optical switch were suggested for the routing of electromagnetic energy on the nanoscale. ${ }^{12}$ Functional macroscopic analogues to such network structures operating in the microwave regime have been demonstrated. ${ }^{13}$

Up to this point, all experimental investigations of plasmon waveguide structures consisting of closely spaced noble

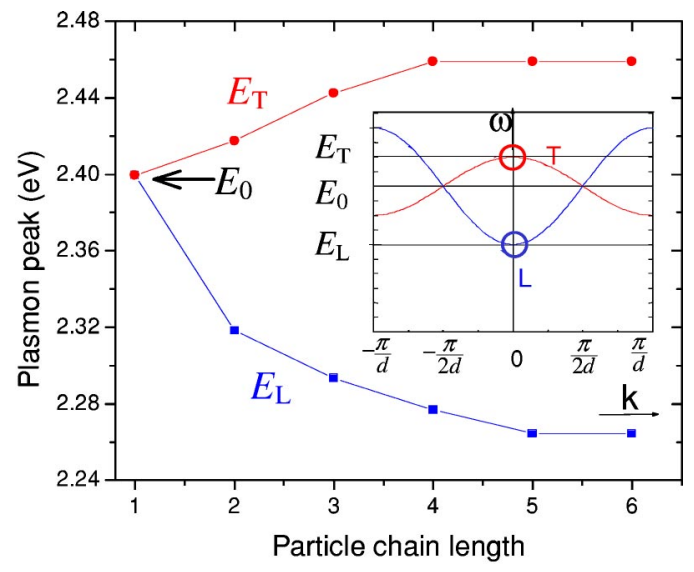

FIG. 1. (Color online) Resonance energies for the longitudinal ( $E_{L}$, squares) and transverse $\left(E_{T}\right.$, circles) collective plasmon modes of Au nanoparticle arrays of different lengths with an interparticle spacing of $3 R$ as obtained by finite-difference time-domain simulations. The inset shows the dispersion relation $\omega(\mathbf{k})$ for this geometry calculated using a point-dipole model (Ref. 11), with the collective modes marked by circles. 
metal nanoparticles have focused on the collective (in-phase) excitations of the long-wavelength mode (wave vector $\mathbf{k}$ $=0) .{ }^{7,8,14}$ Although electromagnetic energy cannot be transported using only this mode, we have shown that far-field measurements of the $\mathbf{k}=0$ mode in combination with the point-dipole model can be used to extract the maximum group velocities. ${ }^{7}$ However, there has been no direct demonstration of pulse propagation in plasmon waveguides, either in experiments or simulations.

In this paper, we demonstrate optical pulse propagation in plasmon waveguides consisting of Au nanoparticles using finite-difference time-domain (FDTD) simulations. The predictions of the point-dipole model for the dispersion relation and the pulse group velocities for energy transport for plasmon waveguides consisting of spherical nanoparticles are confirmed. We also show that local excitation of transverse plasmon modes leads to antiparallel group and phase velocities. Extending our analysis to spheroidal particles with an aspect ratio of 3:1 we demonstrate the ability to tune and improve the guiding parameters of plasmon waveguides.

FDTD simulations that solve the full set of Maxwell's equations on a grid are a powerful method to model lightmatter interactions in complex systems. ${ }^{15}$ We have previously shown that using FDTD simulations we can accurately reproduce spectroscopic properties of plasmon waveguides. ${ }^{7,8}$ Our calculations model plasmon waveguides consisting of up to nine periodically spaced Au nanoparticles with a diameter $D=50 \mathrm{~nm}$ and a center-to-center spacing $d$ $=75 \mathrm{~nm}$. The simulation volume measures $1000 \times 400$ $\times 400 \mathrm{~nm}^{3}$. The particles are surrounded by a medium with a refractive index $n=1$ (vacuum). The material response of $\mathrm{Au}$ is modeled using a Drude model with a bulk plasmon frequency $\omega_{p}=6.79 \times 10^{15} \mathrm{rad} / \mathrm{s}$ and an electron relaxation time $\tau_{\text {Drude }}=4 \mathrm{fs}$ in order to closely match the bulk dielectric function of $\mathrm{Au}$ (Ref. 16) in the vicinity of the single particle resonance $E_{0}$.

In order to determine the collective resonance energies $E_{L, T}$ of the particle chains, the simulation volume is initialized with an electrostatic field chosen to resemble the $\mathbf{k}=0$ mode profile polarized either parallel ( $L$ modes) or perpendicular ( $T$ modes) to the chain direction. The field is then allowed to evolve for $35 \mathrm{fs}$, and its time dependence is analyzed to determine the resonance energies as described in detail elsewhere. ${ }^{8}$ Figure 1 shows the energies $E_{L, T}$ of the collective $\mathbf{k}=0$ modes for plasmon waveguides composed of 1 to $6 \mathrm{Au}$ nanospheres for both the longitudinal polarization $L$ (squares) and transverse polarization $T$ (circles). The peak splitting $\Delta E$ between the $L$ and $T$ modes increases with particle chain length and saturates at $\Delta E=200 \mathrm{meV}$ for chain lengths of five particles. Similar trends were observed in experiments using far-field spectroscopy of plasmon waveguides fabricated using electron beam lithography on ITO coated glass slides. ${ }^{7,8}$ The peak splitting and the known functional form of the point-dipole dispersion relation $\omega(\mathbf{k})$ allow for the calculation of the maximum group velocities $\nu_{g}$ and the energy decay lengths $\alpha$ of the $\mathbf{k}=\pi / 2 d$ modes. ${ }^{7,8}$ For a peak splitting of $200 \mathrm{meV}$, we find $\nu_{g L}=1.6 \times 10^{7} \mathrm{~m} / \mathrm{s}$ for longitudinal and $\nu_{g T}=6.6 \times 10^{6} \mathrm{~m} / \mathrm{s}$ for transverse excitations. Assuming a plasmon decay time $\tau=4$ fs for a single

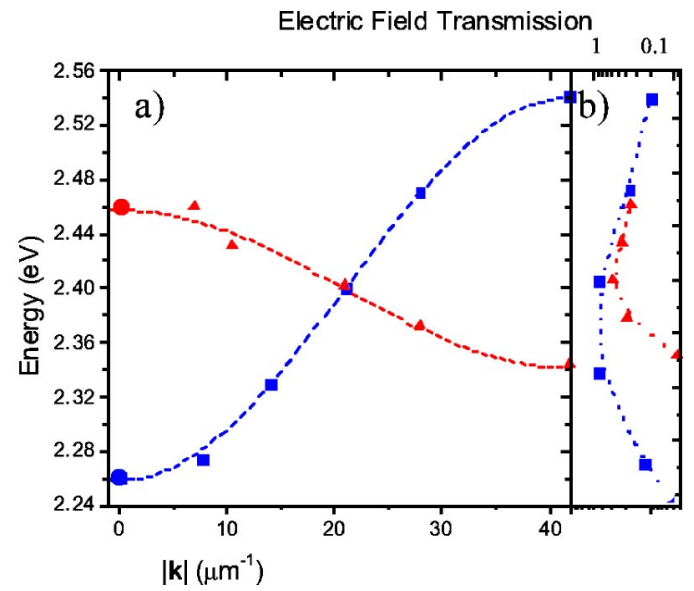

FIG. 2. (Color online) (a) Dispersion relation $\omega(\mathbf{k})$ for plasmon waveguides consisting of spherical particles for both longitudinal (squares) and transverse (triangles) modes obtained by finitedifference time-domain calculations. Also shown are the positions of the $\mathbf{k}=0$ modes (circles) obtained from in-phase excitations and the functional dependence predicted by the point-dipole model (Ref. 11) (dotted lines). (b) For both longitudinal (squares) and transverse (triangles) modes the transmitted electric field (shown on the top axis normalized to the field inside the first particle) in a selected particle is highest when the first particle is excited at the band center.

nanoparticle ${ }^{3}$ the corresponding energy decay lengths are $\alpha_{L}=3 \mathrm{~dB} / 50 \mathrm{~nm}$ and $\alpha_{T}=3 \mathrm{~dB} / 20 \mathrm{~nm}$.

The obtained values for $\nu_{g}$ and $\alpha$ rely on the analytical but approximate point-dipole model of plasmon waveguides. In order to determine the functional form of the dispersion relation $\omega(\mathbf{k})$ with a more realistic model, we simulated the propagation of electromagnetic energy through plasmon waveguide structures using FDTD calculations. The waveguides were excited with an oscillating point-dipole placed at a distance of $d=75 \mathrm{~nm}$ to the center of the first nanoparticle of the waveguide. In consecutive runs, the point-dipole source was driven continuously at various frequencies in the vicinity of $E_{0}$. The field distribution along the nanoparticle chain structure was then analyzed to determine the wave vector $\mathbf{k}$ of the propagating waves. Figure 2(a) shows the thus computed dispersion relation for both longitudinal (squares) and transverse (triangles) excitations, as well as the results for the $\mathbf{k}=0$ modes obtained by plane wave excitation (circles). The dotted lines indicate the dispersion relation calculated using the point-dipole model. The obtained $\omega(\mathbf{k})$ data are in excellent agreement with the predictions from the point-dipole model despite the limitations of the latter. Figure 2(b) shows the electric field amplitude at the center of the last nanoparticle for longitudinal excitations (squares). Note that the waveguide loss is minimum at the center of the dispersion band, as expected since the group velocity is maximum at this point. ${ }^{11}$ The corresponding field amplitudes for transverse modes (triangles) were monitored in particle 2 because of the relatively higher loss under transverse excitations, which together with the reflections makes a monitoring at the last particle difficult. 

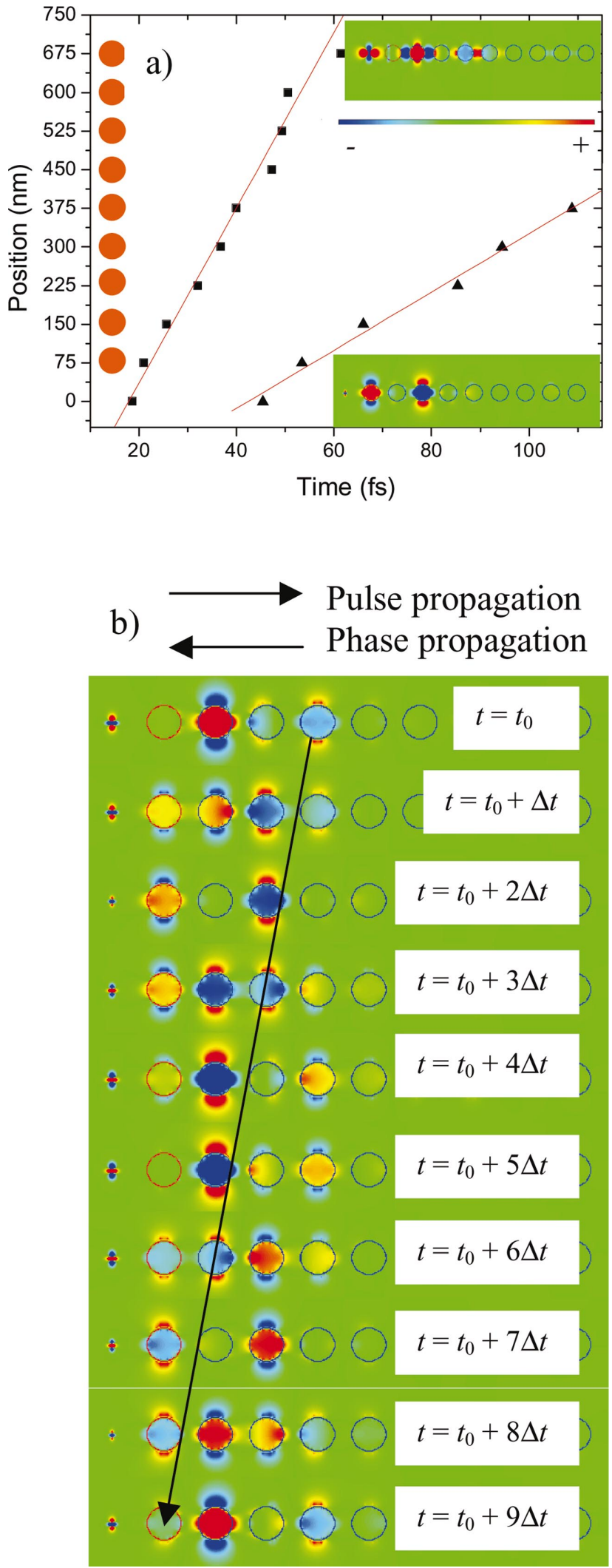

The previous analysis has produced an accurate description of the dispersion relation, but provides no direct evidence for the possibility of information transport using optical pulses. We have therefore also examined the propagation of pulses through these plasmon waveguides by driving the
FIG. 3. (Color) (a) Pulse peak positions over time in a plasmon waveguide consisting of spherical particles for both longitudinal (black squares) and transverse (black triangles) polarization. The spheres along the ordinate indicate the position of the $\mathrm{Au}$ nanoparticles. Snapshots of the $x(y)$ component of the electric field in the xy plane for longitudinal (transverse) polarization are shown in the upper (lower) inset. (b) Time snapshots of the electric field for transverse pulse propagation showing a negative phase velocity with an antiparallel orientation of the phase and group velocities. local dipole source with a pulse centered at the resonance energy $E_{0}=2.4 \mathrm{eV}$ corresponding to the $\mathbf{k}=\pi / 2 d$ modes with the highest group velocities. The width of the pulses was chosen to be $95 \%$ of the bandwidth of the dispersion relation for either polarization in order to keep the pulse 


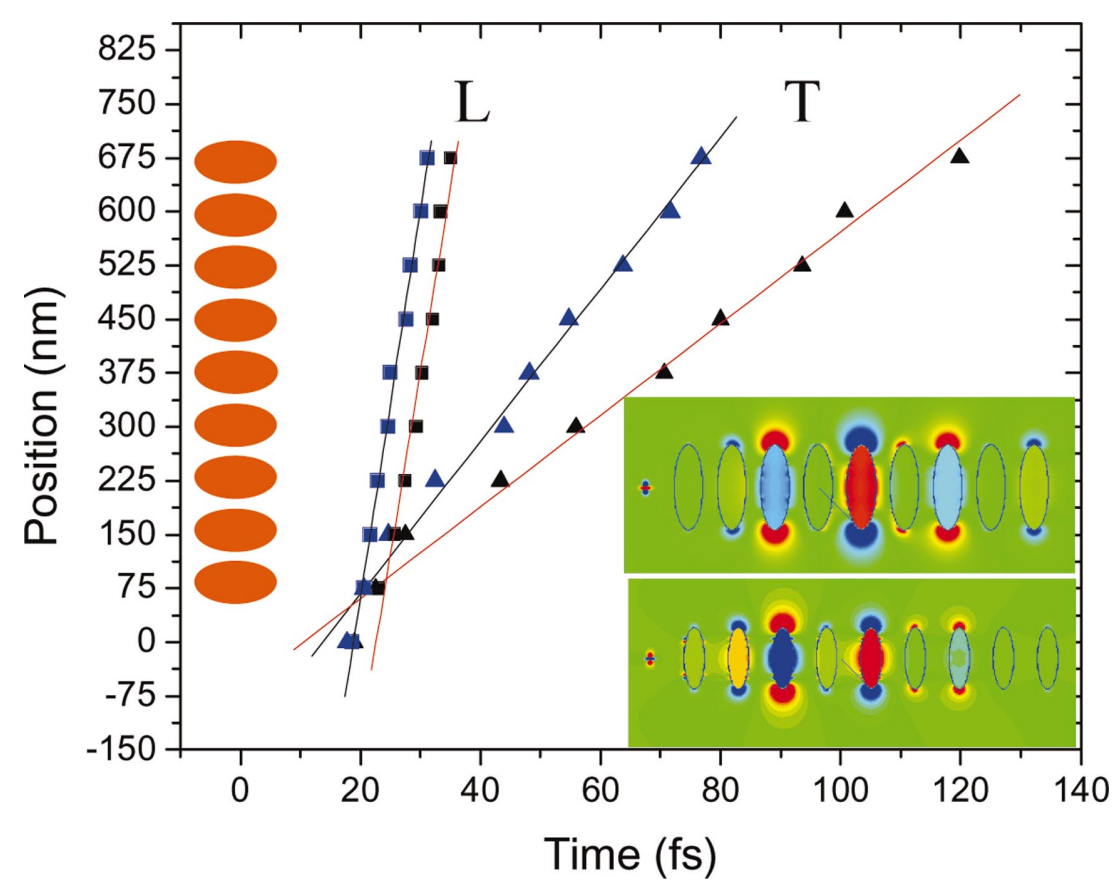

FIG. 4. (Color) Pulse peak positions over time in plasmon waveguides consisting of spheroidal particles with an aspect ratio 3:1 for both longitudinal (squares) and transverse (triangles) polarizations. Shown are data for particles with the same volume as the spherical particles of Fig. 3 (case I, black datapoints) and for particles with an increased volume but the same short axis and surface-to-surface spacing (case II, blue datapoints). The spheroids along the ordinate indicate the position of the Au nanoparticles. The insets show field distributions of transverse pulse propagation through the waveguides for case I (lower inset) and case II (upper inset).

length (30 fs) well within the total simulation time of $125 \mathrm{fs}$. The upper inset of Fig. 3(a) shows a snapshot of the $x$-component of the electric field in the $x y$-plane for longitudinal polarization on a linear color scale. The periodicity of the field distribution along the chain confirms that the pulse is centered around the $\mathbf{k}=\pi / 2 d$ mode corresponding to a wavelength of 4 particle spacings. An analogous snapshot for the $y$-component of the electric field for transverse polarization is shown in the lower inset. Movies of the pulse propagation can be viewed online. ${ }^{17}$ The main part of Fig. 3(a) shows the pulse position, defined as the location of maximum field amplitude, over time for both longitudinal (black squares) and transverse (black triangles) excitation. Linear fits of the datasets yield values for the group velocities of $\nu_{g L}^{*}=1.7 \times 10^{7} \mathrm{~m} / \mathrm{s}$ and $\nu_{g T}^{*}=5.7 \times 10^{6} \mathrm{~m} / \mathrm{s}$, in good agreement with the estimates $\nu_{g L}$ and $\nu_{g T}$ from the point-dipole model $\left(\nu_{g L}=0.94 \nu_{g L}^{*}\right.$ and $\left.\nu_{g T}=1.15 \nu_{g T}^{*}\right)$. The energy decay lengths obtained from the pulse propagation simulations are $\alpha_{L}^{*}=3 \mathrm{~dB} / 140 \mathrm{~nm}$ and $\alpha_{T}^{*}=3 \mathrm{~dB} / 43 \mathrm{~nm}$, respectively. Both are between a factor 2-3 higher than the energy decay lengths calculated using the point dipole model with a plasmon decay time $\tau=4 \mathrm{fs}$. This discrepancy may be due to the finite size of our waveguide, resulting in a discrete spectrum of allowed modes. Nevertheless, these FDTD calculations quantitatively confirm the possibility of using plasmon waveguides for information transport with $\nu_{g}>0.01 c$.

The FDTD calculations clearly show the occurrence of negative phase velocities in these plasmon waveguides when excited in a transverse mode. For the transverse pulse, the group velocity and thus the direction of energy propagation and the phase velocity of the individual wave components are anti-parallel. This is illustrated in Fig. 3(b), which shows ten electric field snapshots spaced $\Delta t=0.166$ fs $(\approx 10 \%$ of a cycle at $E_{0}$ ) apart. The wave packet is seen to move away from the source while the individual phase fronts travel towards the source, ${ }^{17}$ since for transverse modes a positive group velocity, required for causality, occurs at negative wavevectors $\mathbf{k}$, as seen in the dispersion relation depicted in the inset of Fig. 1. Plasmon waveguides could thus serve as a relatively simple model system for the investigation of negative phase velocity systems and be of value in the present discussions regarding the unique properties of these systems. $^{18,19}$

The previous analysis dealt exclusively with spherical particles. Recently, it was shown that rod-shaped nanoparticles with their long axis oriented perpendicular to the waveguide chain axis show an increased inter-particle coupling compared to spherical particles that have the same particle volume and center-to-center spacing. ${ }^{8}$ Additionally, gold nanorods with an aspect ratio of 3:1 were also shown to exhibit an increase in plasmon decay time by about a factor $2-3$ due to a resonance shift away from the interband transition edge. ${ }^{20}$ In order to analyze the effects of the particle shape on the group velocities for pulse propagation, we performed FDTD simulations with pulsed local excitations as described above on two plasmon waveguides consisting of spheroidal particles with an aspect ratio of 3:1 and their long axes oriented perpendicular to the waveguide axis. In the first case, the spheroids were chosen to be of the same volume and center-to-center spacing $d=75 \mathrm{~nm}$ as the 50-nm Au spheres described above, corresponding to a short axis $a=34.7 \mathrm{~nm}$ and a long axis $c=104 \mathrm{~nm}$. The lower inset of Fig. 4 shows 
a snapshot of the $y$ component of the electric field in the $x y$ plane for the propagation of a transverse pulse centered at the resonance frequency $E_{0}$ for this system, showing the $\mathbf{k}$ $=\pi / 2 d$ periodicity. The main part of Fig. 4 shows the position of the propagating pulse peaks for both transverse polarization (electric field polarized along the long spheroid axis, black triangles) and longitudinal polarization (electric field polarized along the short spheroid axis, black squares). Also shown are linear fits to the datasets, corresponding to group velocities $\nu_{3: 1, T}=6.4 \times 10^{6} \mathrm{~m} / \mathrm{s}$ and $\nu_{3: 1, L}=5.1$ $\times 10^{7} \mathrm{~m} / \mathrm{s}$. These values are indeed larger than observed for spherical particles. For the transverse mode, the relative increase in the group velocity compared to the case of spherical particles is $12 \%$ only, even though the bandwidth obtained from the $\mathbf{k}=0$ in-phase calculations increases by about a factor $4.5 .{ }^{8}$ Conversely, for the longitudinal mode the relative increase in group velocity for the longitudinal mode is $300 \%$, while in this case the bandwidth does not change appreciably when going from spherical to spheroidal particles. The fact that $\nu_{g}$ is not linearly related to $\Delta E$ suggests that $\omega(\mathbf{k})$ is strongly affected by the aspect ratio of the nanoparticles. The observed behavior could be caused by the increased radiation damping of transverse modes due to a nonhomogeneous polarization along the long axis which in this geometry is no longer small compared to the wavelength $\lambda$ of the exciting light. ${ }^{21}$

The second case we investigated concerned plasmon waveguides consisting of spheroids with the same center-tocenter spacing but with a larger volume $(a=50 \mathrm{~nm}$ and $c$ $=150 \mathrm{~nm}$ ) than the 50-nm-diameter nanospheres. Note that these structures show an interparticle surface-to-surface spacing of $25 \mathrm{~nm}$, at the limit of current electron beam lithography fabrication techniques. The results from FDTD calculations are also shown in Fig. 4. The upper inset of Fig. 4 shows a snapshot of the $y$ component of the electric field during the propagation of a transverse pulse on this structure. In this case, the maximum group velocities increase to $\nu_{3: 1, T}^{*}=1.06 \times 10^{7} \mathrm{~m} / \mathrm{s} \quad$ (blue triangles) and $\nu_{3: 1, L}^{*}=5.4$ $\times 10^{7} \mathrm{~m} / \mathrm{s}$ (blue squares). The waveguide properties can thus be optimized by tuning the particle geometry.

In summary, FDTD simulations show that plasmon waveguides consisting of closely spaced $\mathrm{Au}$ nanoparticles allow for optical pulse propagation below the diffraction limit. The shape of the dispersion relation $\omega(\mathbf{k})$ for spherical particles obtained from FDTD calculations closely agrees with that predicted by a point-dipole calculation. ${ }^{11}$ For spherical particles, the group velocities are about $0.02 c-0.06 c$ depending on polarization. The guiding properties can be optimized by changes in particle shape and spacing, resulting in group velocities of up to $0.2 c$ for geometries that can be achieved using current fabrication techniques such as electron beam lithography. Plasmon waveguides thus have the potential to be applied in future highly integrated optical circuits, to enable confinement and transport of electromagnetic energy below the diffraction limit, and can be used as a model system for the study of light-matter interactions on the nanoscale.

This work was sponsored by the NSF Grant No. ECS0103543 and the Center for Science and Engineering of Materials at Caltech and the Air Force Office of Scientific Research.
*Email address: stmaier@caltech.edu

${ }^{1}$ U. Kreibig and M. Vollmer, Optical Properties of Metal Clusters (Springer-Verlag, Berlin, 1994).

${ }^{2}$ C. Bohren and D. Huffman, Absorption and Scattering of Light by Small Particles (Wiley, New York, 1983).

${ }^{3}$ T. Klar, M. Perner, S. Grosse, G. von Plessen, W. Spirkl, and J. Feldmann, Phys. Rev. Lett. 80, 4249 (1998).

${ }^{4}$ G. Mie, Ann. Phys. (N.Y.) 25, 377 (1908).

${ }^{5}$ B. Lamprecht, G. Schider, R. T. Lechner, H. Ditlbacher, J. R. Krenn, A. Leitner, and F. R. Aussenegg, Phys. Rev. Lett. 84, 4721 (2000).

${ }^{6}$ S. Linden, J. Kuhl, and H. Giessen, Phys. Rev. Lett. 86, 4688 (2001).

${ }^{7}$ S. A. Maier, M. L. Brongersma, P. G. Kik, and H. A. Atwater, Phys. Rev. B 65, 193408 (2002).

${ }^{8}$ S. A. Maier, P. G. Kik, and H. A. Atwater, Appl. Phys. Lett. 81, 1714 (2002).

${ }^{9}$ S. I. Bozhevolnyi, J. Erland, K. Leosson, P. M. W. Skovgaard, and J. M. Hvam, Phys. Rev. Lett. 86, 3008 (2001).

${ }^{10}$ M. Quinten, A. Leitner, J. R. Krenn, and F. R. Aussenegg, Opt. Lett. 23, 1331 (1998).
${ }^{11}$ M. L. Brongersma, J. W. Hartman, and H. A. Atwater, Phys. Rev. B 62, R16356 (2000).

${ }^{12}$ S. A. Maier, M. L. Brongersma, P. G. Kik, S. Meltzer, A. A. G. Requicha, and H. A. Atwater, Adv. Mater. 13, 1501 (2001).

${ }^{13}$ S. A. Maier, M. L. Brongersma, and H. A. Atwater, Appl. Phys. Lett. 78, 16 (2001).

${ }^{14}$ J. R. Krenn, A. Dereux, J. C. Weeber, E. Bourillot, Y. Lacroute, J. P. Goudonnet, G. Schider, W. Gotschy, A. Leitner, F. R. Aussenegg, and C. Girard, Phys. Rev. Lett. 82, 2590 (1999).

${ }^{15}$ For a selected review see K. L. Shlager and J. B. Schneider, IEEE Antennas Propag. Mag. 37, 39 (1995).

${ }^{16}$ P. B. Johnson and R. W. Christy, Phys. Rev. B 6, 4370 (1972).

${ }^{17}$ Movies showing the evolving pulse fields can be viewed at http:// daedalus.caltech.edu/research/nanophotonics.html.

${ }^{18}$ D. R. Smith and N. Kroll, Phys. Rev. Lett. 85, 2933 (2000).

${ }^{19}$ J. B. Pendry, Phys. Rev. Lett. 85, 3966 (2001).

${ }^{20}$ C. Sönnichsen, T. Franzl, T. Wilk, G. von Plessen, J. Feldmann, O. Wilson, and P. Mulvaney, Phys. Rev. Lett. 88, 077402 (2002).

${ }^{21}$ M. Scharte, R. Porath, T. Ohms, M. Aeschlimann, J. R. Krenn, H. Ditlbacher, F. R. Aussenegg, and A. Liebsch, Appl. Phys. B: Lasers Opt. 73, 305 (2001). 